\title{
DIE GEHEIMNISSE RUND UM DIE KAWASAKI-KRANKHEIT: EINE LITERATURÜBERSICHT
}

\section{ORIGINALER ARTIKEL}

ROSSI, Karoline ${ }^{1}$, MOREIRA, Danilo José Silva ${ }^{2}$, FONSECA, Juliana Brito da ${ }^{3}$, VASCONCELOS, Suzana dos Santos ${ }^{4}$, OLIVEIRA, Vinicius Faustino Lima de ${ }^{5}$, DIAS, Claudio Alberto Gellis de Mattos $^{6}$, OLIVEIRA, Euzébio de ${ }^{7}$, DENDASCK, Carla Viana $^{8}$, ARAÚJJ, Maria Helena Mendonça de ${ }^{9}$ FECURY, Amanda Alves ${ }^{10}$

ROSSI, Karoline. Et al. Die Geheimnisse rund um die Kawasaki-Krankheit: Eine Literaturübersicht. Revista Científica Multidisciplinar Núcleo do Conhecimento. Jahrgang 06, Ed. 04, Vol. 05, S. 52-64. April 2021. ISSN: 2448-0959, Zugangslink: https://www.nucleodoconhecimento.com.br/gesundheit/kawasaki-krankheit, DOI: 10.32749/nucleodoconhecimento.com.br/gesundheit/kawasaki-krankheit

\section{ZUSAMMENFASSUNG}

Kawasaki-Krankheit (DK) oder mucokutane Lymphknoten-Syndrom ist eine systemische Vaskulitis, die vor allem Kinder unter fünf Jahren mit asiatischer Abstammung betrifft, aber auch andere Altersgruppen erreichen kann, sowie jede

\footnotetext{
${ }^{1}$ Medizinische Akademiker. Föderale Universität von Amapá (UNIFAP).

${ }^{2}$ Mediziner. Föderale Universität von Amapá (UNIFAP).

${ }^{3}$ Medizinische Akademiker. Föderale Universität von Amapá (UNIFAP).

${ }^{4}$ Medizinische Akademiker. Föderale Universität von Amapá (UNIFAP).

${ }^{5}$ Mediziner. Föderale Universität von Amapá (UNIFAP).

${ }^{6}$ Biologe, PhD in Theorie- und Verhaltensforschung, Professor und Forscher des Studiengangs Chemie des Instituts für Grund-, Technik- und Technologiepädagogik (IFAP).

${ }^{7}$ Biologe, PhD in Tropenkrankheiten, Professor und Forscher des Physical Education Course, Federal University of Pará (UFPA).

${ }^{8}$ Theologe, PhD in klinischer Psychoanalyse. Er hat 15 Jahre mit Scientific Methodology (Research Method) in der Orientierung der wissenschaftlichen Produktion von Master- und Doktoranden gearbeitet. Spezialist für Marktforschung und Forschung mit Schwerpunkt Gesundheit.

${ }^{9}$ Doktor, Master in Lehr- und Gesundheitswissenschaften, Professor und Forscher des Medical Course of Macapa Campus, Federal University of Amapá (UNIFAP).

${ }^{10}$ Biomedizin, PhD in Tropenkrankheiten, Professor und Forscher des Medical Course of Macapa Campus, Federal University of Amapá (UNIFAP).
}

RC: 82508

Disponível em: https://www.nucleodoconhecimento.com.br/gesundheit/kawasaki- 
andere Rasse. Das klinische Bild von DK hat drei Stadien: akutes febriles Stadium, in dem Bindehautverstopfung, Mundschleimhaut, Erythem, Abplatzung, polymorpher Hautausschlag und spätere zervikale Lymphadenopathie als Hauptsymptome auftreten; das subakute Stadium, das am Ende des Fiebers auftritt, und führt zum Auftreten von Hautabblättern in den Gliedmaßen, Arthritis, Arthralgie und Thrombozytose und schließlich das Stadium der Rekonvaleszenz, das entsteht, wenn die Symptome fast verflüchtigt sind und bis zu ihrer Normalisierung anhalten. Die am häufigsten verwendete Behandlung erfolgt aus der Verabreichung von intravenösem Immunglobulin, das für eine bessere Prognose der Pathologie frühzeitig eingeleitet werden sollte.

Schlüsselwörter: Mukokutanes Lymphknotensyndrom, Vaskulitis, Arteritis, Fieber, Kawasaki.

\section{EINLEITUNG}

Die Kawasaki-Krankheit (DK) ist eine systemische Vaskulitis, die kleine und mittlere Gefäße betrifft und hauptsächlich Kinder unter 5 Jahren betrifft. Dies ist einer der Gründe für Herzerkrankungen im Kindesalter (HUANG et al., 2015; MAGALHÃES, 2008). Die Ätiologie von DK, dh die Erreger dieser Krankheit, sind nicht bekannt. Einige klinische und epidemiologische Aspekte legen jedoch mögliche infektiöse Gründe nahe (FERRONATO et al., 2010).

DK ist die zweithäufigste Vaskulitis im pädiatrischen Alter, von der vor allem Kinder unter einem halben Jahrzehnt betroffen sind (90\%). Es ist ungewöhnlich bei Kindern unter 6 Monaten und älter als 8 Jahre, die jedoch anfälliger für die Entwicklung von koronaren Aneurysmen sind. Auf globaler Ebene gibt es eine Veränderung des Auftretens, wobei Japan das am stärksten betroffene Land ist, mit einer jährlichen Inzidenz von 110 bis 150 Fällen pro 100.000 Kinder unter 5 Jahren. Unter Berücksichtigung der Berechnung der Inzidenzraten bei 100.000 Kindern unter 5 Jahren in den Vereinigten Staaten von Amerika (USA) ist DK die häufigste Ursache

$\mathrm{RC}: 82508$

Disponível em: https://www.nucleodoconhecimento.com.br/gesundheit/kawasakikrankheit 
für im Kindesalter erworbene Herzerkrankungen, die von 9,1 bis 32,5 Fällen reicht und häufiger bei nachkommen asiatischer und pazifischer Inseln auftritt $(32,5)$, zwischengeschalteter Afroamerikaner (16,9) und Hispanics $(11,1)$ und bei Kaukasiern (9,1) (ALMEIDA et al., 2010; CASTRO; URBANO; COSTA, 2009; RODRIGUES et al, 2017).

DK hat eine höhere Prävalenz in der asiatischen Bevölkerung, mit Schwerpunkt auf Japanisch, die vor allem Kinder betrifft. Es gibt einen Bericht über eine jährliche Inzidenz von bis zu 100 Fällen pro 100.000 Einwohner in Japan und Korea, während es im kaukasischen Rasse zwischen 6 und 10 Fällen pro 100.000 Einwohner unter einem halben Jahrzehnt liegt. In Lateinamerika gibt es schätzungsweise 3 Fälle pro 100.000 Einwohner, was eine geringere Häufigkeit dieser Pathologie bei nichtasiatischen Populationen, die nicht von Asiaten abstammen, besagt (SCARDINA et al., 2007). In Bezug auf die mit DK assoziierte Sterblichkeitsrate ist sie sehr niedrig, da sie unter $0,1 \%$ liegt (BARCA et al., 2019).

Die Inzidenz von DK variiert geografisch in der Welt, wobei sie bei japanischen Nachkommen häufiger ist, was auf eine genetische Veranlagung der Krankheit hindeutet, zusätzlich zu möglichen causatous-Erregern, die mit dem geografischen Standort in Verbindung gebracht werden können, wie Bakterien (CASTRO; URBANO; COSTA, 2009; RODRIGUES et al, 2017).

Die saisonale Veränderung der Inzidenz von DK ist gut anerkannt, aber die Raten ändern sich zwischen den Ländern. Im Vereinigten Königreich, in Australien und in den USA gibt es im Winter und Frühjahr eine höhere Zahl von Fällen. In China und Korea, im Frühjahr und Sommer. In den USA und uk korreliert der Anstieg der Raten eher mit der Variation der Regenperioden als mit temperaturschwankungen. In Brasilien zeigte eine Studie mit 70 DK-Patienten eine höhere Aufzeichnung der Krankheit, die mit Perioden höherer Inzidenz von Infektionskrankheiten von Mai bis Juni (Beginn der Dürre) und November und Dezember (frühe Regenfälle) zusammenfällt (MAGALHÃES; ALVES, 2017).

RC: 82508

Disponível em: https://www.nucleodoconhecimento.com.br/gesundheit/kawasakikrankheit 
Eine Person mit DK entwickelt symptomatologische Eigenschaften, die zur Darstellung eines klinischen Bildes führen, das aus der Reihe von Anzeichen und Symptomen besteht, die vom Patienten manifestiert werden (KAYIRAN; DINDAR; GURAKAN, 2010). So ist das klinische Bild von DK durch zervikale Lymphadenopathie, Fieber, Tonsillitis, Schwellung der Gliedmaßen, Hautausschlag, Juckreiz und Abplatzen gekennzeichnet (ATIK, 2007; SCARDINA et al., 2007). Darüber hinaus kann DK generalisierte Entzündungen verursachen, die einige Organe verursachen entzündliche Kardiomyopathie, sterile Meningitis, Hepatitis, Adenolinfit, Perikarditis und Angitis (CASTRO; URBANO; COSTA, 2009).

Castro, Urbano und Costa (2009) weisen auf einen Zusammenhang zwischen Vaskulitis durch DK und der Beteiligung einiger Organe hin, die aus einer Gruppierung von Geweben bestehen, die für die Erhaltung des Organismus verantwortlich sind.

DK wurde erstmals 1967 vom japanischen Arzt Tomisaku Kawasaki berichtet, der es als "mukokutanes Lymphknotensyndrom" definierte. In diesem Zeitraum wurden in den Jahren 1961 bis 196750 Fälle gemeldet. In Japan ist das Vorkommen höher, und die globale Verteilung hat ihre variable Prävalenz (ALMEIDA et al., 2010; SCARDINA et al., 2007; MAGALHÃES; ALVES, 2017).

Im aktuellen Szenario wurden bereits einige epidemiologische Daten über DK erstellt, die eine genauere Analyse der Faktoren ermöglichen, die bei der Verbreitung und Ausbreitung von Krankheiten eingreifen. Viele Studien wurden durchgeführt, um die Pathophysiologie der Krankheit zu verstehen, die aus einer Reihe von abnormalen Veränderungen im Körper verursacht besteht (CASTRO; URBANO; COSTA, 2009).

In einer Studie wurden Hinweise auf DK und den Fortschritt anderer Komorbiditäten gefunden, was auf die Tatsache verweist, dass zwei oder mehr Krankheiten

$\mathrm{RC}: 82508$

Disponível em: https://www.nucleodoconhecimento.com.br/gesundheit/kawasakikrankheit 
gleichzeitig in einem Individuum sind, so dass die Komplikationen von DK die ungünstige Entwicklung dieser Assoziation wäre (SCARDINA et al., 2007).

Um die Versorgung und Lebensqualität des Patienten mit DK zu verbessern, wurden Behandlungen entwickelt, die aus Möglichkeiten der Pflege eines Patienten bestehen (CASTRO; URBANO; COSTA, 2009).

\section{ZIELE}

Erläutern Sie die möglichen causatous Enzius-Erreger, die Pathophysiologie, die Hauptorgane und anatomischen Strukturen, die von der Kawasaki-Krankheit (DK) betroffen sind.

Zur Überprüfung der Hauptmerkmale des klinischen Bildes einer Person für die Diagnose, der wichtigsten damit verbundenen Komplikationen und der Behandlungen, die für Patienten mit DK zur Verfügung stehen.

\section{METHODE}

Dies ist eine bibliographische Forschung, die in den Datenbanken Scielo, Brazilian Digital Library of Thesen and Dissertations, LILACS und PubMed unter Verwendung von Schlüsselwörtern oder deren Assoziationen durchgeführt wird: KawasakiKawasaki-Krankheit.

Die bei der Erstellung der Recherchen verwendeten Inklusionsparameter waren die umfassende Online-Verfügbarkeit, der direkte Ansatz zur Kawasaki-Krankheit (DK) oder in einem für diese Krankheit relevanten Aspekt. In der Scielo-Datenbank gab es Textfilter der in Englisch und Portugiesisch geschriebenen Produktionen, in den anderen Datenbanken wurde dieses Kriterium nicht verwendet. Die angewendeten Ausschlussparameter wurden dupliziert und vor dem Jahr 2000 ausgeführt.

RC: 82508

Disponível em: https://www.nucleodoconhecimento.com.br/gesundheit/kawasakikrankheit 
In den genannten Datenbanken wurde eine Analyse durchgeführt, um die Artikel über DK in der Literatur aufzulisten. Aus einer früheren Lektüre der Titel und Zusammenfassungen der gefundenen wissenschaftlichen Produktionen wurden Studien verworfen, die nicht dem entsprachen, was diese Rezension darstellen sollte. Die vollständigen Texte, die sich durch die vorherigen Phasen hindurchzogen, wurden gelesen und für diese Überprüfung diejenigen ausgewählt, die relevante Aspekte von DK behandelten.

\section{ERGENISSBE}

\section{MÖGLICHE CAUSATOUS AGENTS OF DK}

Die Ursache der Kawasaki-Krankheit ist noch unbekannt. Seine klinischen und epidemiologischen Eigenschaften nähren jedoch die Hypothese, dass ein Infektiöser die auslösende Ursache ist, was Veränderungen in den Zellen, Geweben und Organen von Individuen mit genetischer Veranlagung hervorruft. Diese Hypothese ist die wahrscheinlichste, aber sie hat immer noch keinen Beweis (CASTRO; URBANO; COSTA, 2009).

Eine der Theorien ist die des Coronavirus NL-63, das mittels einer Polymerase Chain Reaction (PCR) in Atemwegsflüssigkeiten von 11 Patienten mit DK und nur bei nur einem der 22 Kontrollpatienten nachgewiesen wurde. Weitere 5 spätere Studien fanden jedoch kein Coronavirus in Atemwegs- oder Nasopharitgewebeproben von Patienten, was es unmöglich machte, dass dieses Virus die auslösende Ursache war (CASTRO; URBANO; COSTA, 2009).

Eine andere Theorie ist die der immunologischen Stimulation durch bakterielle Superantigene wie Staphylokokken und Streptokokkentoxine. Eine dritte Theorie ist immunologisch, die vorschlägt, dass DK durch eine abnorme Immunantwort bei genetisch veranlagten Individuen verursacht werden soll (MAGALHÃES; ALVES, 2017).

RC: 82508

Disponível em: https://www.nucleodoconhecimento.com.br/gesundheit/kawasakikrankheit 


\section{PHYSIOPATHOLOGIE VON DK}

Die in DK gefundenen Veränderungen, insbesondere histologische, charakterisieren ein Bild der generalisierten systemischen Angiitis, die hauptsächlich Gefäße von medianem Kaliber, insbesondere die Herzkranzgefäße, betrifft. In mehreren Organen können berüchtigte Veränderungen beobachtet werden, wie z. B. im Myokard, im Perikard, in den Blutgefäßen, in den Hirnhäuschen, in der Lunge, in den Lymphknoten und in der Leber (CASTRO; URBANO; COSTA, 2009).

\section{WICHTIGSTE ANATOMISCHE ORGANE UND STRUKTUREN, DIE VON DK BETROFFEN SIND}

DK betrifft Organe und Arterien, vor allem Gefäße von Mittelkaliber aus verschiedenen Systemen des menschlichen Körpers, wie Herz-Kreislauf, Lungen, Magen-Darm (Darm und Gallenblase sind die wichtigsten betroffenen Organe) und das zentrale Nervensystem (SNC). Es sollte jedoch die Implikation hervorgehoben werden, die diese Krankheit im Herzen hat, mit der Bildung von koronaren Aneurysmen (CASTRO; URBANO; COSTA, 2009).

Andere Arterien können auch betroffen sein, wie: Aortenaneurysm, mit einer größeren Anzahl von gemeldeten Fällen kompromittieren die Bauchaorta; Axillary Arterie Aneurysmus; brachiocephalische Arterie Aneurysmus; Aneurysmen von iliac Arterien, femoral; Nierenarterieaneurysmus und Augenarterienverschluss (ALVES et al., 2011).

\section{MAIN FEATURES DES KLINISCHEN BILDES EINES INDIVIDUAL FÜR DIE DIAGNOSE VON DK}

Fieber, das die akute Phase der Krankheit markiert, ist in der Regel erhöht (über $39^{\circ} \mathrm{C}$ ) und in Spitzen, vorübergehend abnehmend in der Intensität dauert etwa sieben bis vierzehn Tage, und kann drei und / oder vier Wochen, wenn es keine

RC: 82508

Disponível em: https://www.nucleodoconhecimento.com.br/gesundheit/kawasakikrankheit 
geeignete Behandlung. Der Tag, an dem das Fieber beginnt, gilt als der erste Tag der Krankheit. Jedoch, einige Patienten haben andere klassische Manifestationen vor Fieber, wie: nicht eitrige Konjunktivitis; zervikale Lymphknotenmegalie; Erytheme und/oder Ödeme an Händen und Füßen; Exanthetem escalatiniform, morbiliform oder polymorph; framboesiform Zunge, Erythem und Oropharynx Ödem, Risse und labiale Erythem (CASTRO; URBANO; COSTA, 2009).

Veränderungen in den Extremitäten (Hände und Füße) mit Erytheme und/oder Ödemen treten auch in der akuten Phase auf, und Ödeme sind sehr schmerzhaft und werden mit palmoplantarem Erythem mit Vaskulitis an den Fingerspitzen assoziiert, die nach einem Zeitraum von ein bis zwei Wochen, bereits in der subakuten Phase, beginnt zu schälen, beginnend mit der periunguealen Region im Handschuhfinger (MAGALHÃES; ALVES, 2017).

Nicht exsudative bilaterale Konjunktivitis wirkt sich insbesondere auf die Bulbarkonjunktiva im Vergleich zur palpebralen und tarsalen Bindehaut aus und ist schmerzlos. Manchmal wird es von Iridocyclitis begleitet, einer Entzündung des vorderen Teils des Auges, mit schneller Auflösung und wenig mit Lichtempfindlichkeit verbunden (CASTRO; URBANO; COSTA, 2009).

Polymorpher Hautausschlag ist eine Reihe von Ausschlägen, die in den ersten Tagen der Krankheit auftreten und hauptsächlich den Rumpf und die Extremitäten betreffen, ohne offensichtliche Blasen oder Vesikel. Sie haben eine variable Darstellung (urticariforme Ausschlag, morbiliform, maculopapular) oder diffus, ähnlich Scharlach (ALMEIDA et al., 2010).

Veränderungen in den Lippen und der Mundhöhle 9 von 10 Fällen in der akuten Phase mit intensiver labialer und oropharynxhyperämie, Rissen, trockener Schleimhaut und Blutungen der Lippen. Zungenpapillen können prominent werden und orale Geschwüre mit Oropharynx exudato können manchmal beobachtet werden. In Bezug auf die zervikale Lymphadenomegalie, ein weiteres diagnostisches

RC: 82508

Disponível em: https://www.nucleodoconhecimento.com.br/gesundheit/kawasakikrankheit 
Kriterium, muss der Patient mindestens einen Lymphknoten mit einem Durchmesser größer oder gleich $1,5 \mathrm{~cm}$, häufig einseitig, schmerzhaft und fest, im vorderen Zervixdreieck (ALMEIDA et al., 2010; MAGALHÃES; ALVES, 2017).

\section{DIE MAIN COMPLICATIONS ASSOCIATED MIT DK}

Bei Patienten mit DK werden drei Arten von Vaskulopathien in verschiedenen Momenten in der Entwicklung der Krankheit identifiziert: nekrotisierende Arteritis, subakute/chronische Arteritis und koryptorische myofibroblastische Proliferationsvaskulitis. In der akuten Phase der Krankheit sticht die nekrotisierende Arteritis hervor, die ihren vollständigen pathologischen Prozess in den ersten zwei Wochen nach Beginn des fiebrigen Zustandes hat, insbesondere in den Herzkranzgefäßen, die die Bildung von Riesenaneurysmen bestimmen können, die anfällig für die Entwicklung von Thrombose und Brüchen sind. In der subakuten Phase wird überprüft, dass der Prozess der subakuten Vaskulitis 2 Wochen nach Beginn des Fiebers beginnt und alle Blutgefäße beeinflussen kann, insbesondere das mittlere Segment der mittleren Muskelarterien, wie die Herzkranzgefäße. In der chronischen Phase der Krankheit tritt eine koroxidierte fibroblastische Myoproliferation auf, die mit chronischer subakuter Vaskulitis (MAGALHÃES; ALVES, 2017).

Die schwerwiegendste Komplikation der Krankheit ist die koronare Vaskulitis mit Veränderungen in den Herzkranzgefäßen, von denen 15-20\% der Patienten betroffen sind, die keine Behandlung erhalten. $\mathrm{Zu}$ diesen Veränderungen gehören Aneurysmen, Ekekasien und Steosen der Herzkranzgefäße, die für $2 \%$ Mortalität verantwortlich sind (ALMEIDA et al., 2010).

Im Magen-Darm-System gibt es Erbrechen, Durchfall, Bauchschmerzen und lähmenden Ileus. Zu Beginn des Fiebers sind erbrechendes Erbrechen und Durchfall am häufigsten und am wenigsten häufig Gelbsucht, schmerzhafte Hepatomegalie, Pankreatitis und hydropische Vesikel. In den Atemwegen sind husten, Pneumonitis

$\mathrm{RC}: 82508$

Disponível em: https://www.nucleodoconhecimento.com.br/gesundheit/kawasakikrankheit 
und radiologische Veränderungen am häufigsten. Selten tritt rinorreia (MAGALHÃES; ALVES, 2017).

Im SNC kann sich die Krankheit mit extremer Reizbarkeit in der akuten Phase manifestieren, zusätzlich zu aseptischer Meningitis, Ataxie, Gesichtslähmung und sensorineuralem Hörverlust. Bemerkenswert ist auch das Vorhandensein von Ganglionitis und Neuritis in Schädel- und peripheren Nerven, Endarteritis, Periarteritis, Choriomeningitis und Leptomeningitis, zusätzlich zu Atrophie, Degeneration mit Verlust von Neuronen, marginale und subpendimale Gliose und Bildung von Gliaknollen um Neuronendegenerationen (MAGALHÃES; ALVES, 2017).

Verhaltensänderungen wie Aufmerksamkeitsstörungen, Lernmangel, emotionale Veränderungen (emotionale Labilität, Angst und Nachtterror) und Internalisierungsprobleme (ängstliches, depressives und aggressives Verhalten) wurden in der Literatur beschrieben (ALVES et al., 2011).

\section{BEHANDLUNGEN VERFÜGBAR FÜR INDIVIDUALS AFFECTED VON DK}

Die Therapie für DK hat zwei verschiedene Zwecke, abhängig von der Phase, in der sie sich befindet. In der akuten Phase zielt es darauf ab, die Entzündungsreaktion in der herzkranzigen Herzkranzwand zu reduzieren und Vaskulitis und ihre Folgen (Thrombose und Aneurysmen) zu verhindern. In den subakuten und chronischen Phasen soll Myokardischämie und Infarkt verhindert werden (CASTRO; URBAN; COSTA, 2009).

Intravenöses Immunglobulin (IGEV) ist das wichtigste Arzneimittel, das bei DK verwendet wird, und sein Wirkmechanismus bleibt unbekannt. Es wird in der akuten Phase verwendet, vorzugsweise in den ersten 7 bis 10 Tagen nach Beginn der Erkrankung (MAGALHÃES; ALVES, 2017).

RC: 82508

Disponível em: https://www.nucleodoconhecimento.com.br/gesundheit/kawasakikrankheit 
Die Behandlung besteht in der Verabreichung von Immunglobulin in einer Einzeldosis von $2 \mathrm{~g} / \mathrm{kg}$ über einen variablen Zeitraum von 10 bis 12 Stunden zusammen mit der Verabreichung von Acetylsalicylsäure (AAS) 80 bis 100 $\mathrm{mg} / \mathrm{kg} / \mathrm{Tag}$, oral, 6/6 Stunden. Hochdosierte ASA hat eine entzündungshemmende Wirkung und wirkt in niedrigen Dosen als Thrombozytenaggregationshemmer, so dass die Zeit der Anwendung von AAS in hohen Dosen so lange verbleiben sollte, bis das Kind afebrile ist. Anschließend wird die Dosis auf 3 bis $5 \mathrm{mg} / \mathrm{kg} / \mathrm{Tag}$ reduziert und bei dieser Dosis beibehalten, während der Patient Thrombozytose und/oder koronare Veränderungen vorlegt (MAGALHÃES; ALVES, 2017; RODRIGUES et al., 2017).

Zwischen 1 und 2 von 10 Patienten mit DK reagieren nicht auf die erste Dosis von IGEV, oder mit der Dauerhaftigkeit der fiebrigen Erkrankung oder mit dem Wiedererscheinen davon eineinhalb Tage nach der ersten Infusion. Diese Patienten gelten als refraktär zur Behandlung und sind die Patienten mit dem höchsten Risiko für koronare Veränderungen. In solchen Fällen wird die Verabreichung der zweiten Dosis von IGEV von 2 g/kg in einer Einzeldosis empfohlen. Wenn Fieber 36 Stunden lang anhält, wird empfohlen, eine Pulstherapie mit Methylprednisolon in einer Dosis von $30 \mathrm{mg} / \mathrm{kg} / \mathrm{Tag}$ (maximale Dosis $1 \mathrm{~g} / \mathrm{Tag}$ ), infundiert bei 1 Stunde, 1 Zeit/Tag, für 3 Tage (MAGALHÃES; ALVES, 2017).

Bei der Behandlung von DK in der subakuten und chronischen Phase wird niedrig dosiertes Aspirin (3-5mg/kg/Tag) bei Kindern mit kleinen und mittleren Aneurysmen angewendet. In diesen Phasen zielt die Behandlung darauf ab, Thrombose (Thromboletaktivierung) und Gefäßsthesis zu verhindern. Andere Thrombozytenmittel werden ebenfalls verwendet (Clopidogrel, Ticlopidin, Dipyridamol) und, verbunden mit Aspirin, präsentieren sich als konkreter bei der Blockierung der Thrombozytenaggregation (CASTRO; URBAN; COSTA, 2009).

\section{DISKUSSION}

$\mathrm{RC}: 82508$

Disponível em: https://www.nucleodoconhecimento.com.br/gesundheit/kawasakikrankheit 


\section{KORRELATION ZWISCHEN POSSIBLE CAUSATORS UND DER ENTWICKLUNG VON DK}

Bis heute wurde der Ursprung von DK nicht identifiziert, aber es gibt einige Agenten, von denen angenommen wird, dass sie mit der Entwicklung der Pathologie zusammenhängen. Dies sind Staphylococcus aureus, estreptococos, vírus influenza, morbilivírus, paramixovírus, bunyavírus. Adenovírus und ein neues humanes Coronavirus namens New Haven Coronavirus (Nco-NH) können ebenfalls mit DK in Verbindung gebracht werden, da sie bereits in Atemwegssekrete von Patienten mit der Krankheit identifiziert wurden. Solche Viren und Bakterien könnten auf die Entwicklung von DK wirken, so dass sie zu Daten führen würden, die durch die Krankheit verursacht werden, wie Aortenaneurysmen (BARCA et al., 2019; CASTRO; URBANO; COSTA, 2009).

Es gibt eine Hypothese, die die Existenz von bakteriellen Superantigenen mit der Entwicklung von DK assoziiert. Studien, die diesen Vorschlag analysieren, gehen davon aus, dass das Superantigen durch den Haupthistokompatibilitätskomplex der Klasse II (Klasse II MHC) und den T-Lymphozytenrezeptor an anderen Anleinen als bei gemeinsamen Antigenen an die Antigene binden kann. Dies würde eine immunologische Reaktion mit der Bildung und Freisetzung von proinflammatorischen Zytokinen in übermäßigen Konzentrationen fördern und zur Entwicklung eines entzündlichen Prozesses beitragen, der für DK charakteristisch ist (CASTRO; URBANO; COSTA, 2009).

Einige Indikationen führen zu dem Glauben, dass eine oligoklonale IgA-Antwort bei akuter DK auftritt. Eine von Castro, Urbano und Costa (2009) durchgeführte Studie führte die Analyse der alpha schweren Kette von Immunglobulinen durch, die aus einer großen Menge von Leukozyten gesammelt wurden, die an einer Entzündlichen Reaktionsstelle der arteriellen Wand von Patienten mit DK angesammelt wurden, was zeigte, dass spezifische Immunglobulin-A-Antikörper in der Probe nachgewiesen

RC: 82508

Disponível em: https://www.nucleodoconhecimento.com.br/gesundheit/kawasakikrankheit 
wurden. Dies belegt die Existenz einer Immunantwort im Zusammenhang mit DK (CASTRO; URBANO; COSTA, 2009).

Die Hypothese, dass DK genetische Veranlagung hat, wird durch die hohe Inzidenz unter Asiaten und ihren Rummies verstärkt. Eine Studie zeigte 67 Gene im Zusammenhang mit der Entwicklung der Pathologie, die Beteiligung am Endothel, Lipidstoffwechsel, Aktivierung der immunologischen Mechanismen und Thrombozytenrekrutierung beteiligt, unterstützt die Idee, dass es eine genetische Beziehung für die Entwicklung von DK (CASTRO; URBANO; COSTA, 2009; SOTELO; GONZÁLEZ, 2007).

\section{PHYSIOPATHOLOGIE VON DK}

Vaskulitis beginnt mit dem Auftreten von Schwellungen und Entzündungen, die für einen variablen Zeitraum auftreten können. Neutrophile sind zunächst vorhanden, und bald darauf gibt es eine Dominanz von CD8+ zytotoxischen T-Lymphozyten und IgA-Immunglobulin (CASTRO; URBAN; COSTA, 2009).

In den Herzkranzgefäßen gibt es entzündliche Veränderungen mit dem Vorhandensein von Ödemen und Nekrose von Muskelzellen. Mit diesem Verlust der strukturellen Integrität gibt es die Bildung von Aneurysmen. Darüber hinaus vermehren sich Fibroblasten und bauen sich dann um, was zu einem möglichen Vorhandensein von Susthese, Verkalkungen und Thrombose führt (CASTRO; URBANO; COSTA, 2009; SOTELO; GONZÁLEZ, 2007).

Es ist wichtig zu beachten, dass Entzündlicher Faktor 1 in Allografts in shimnotischen arteriellen Geweben von DK stark erhöht ist. Dieser Entzündungsfaktor 1 des Allografts bindet die Reaktion des Interferontyps I bis zur Aktivierung spezifischer Makrophagen und T-Lymphozyten, die die wahrscheinliche Bedeutung der Interferenz dieser Zellen in der Arteritis durch DK (ROWLEY et al., 2017).

$\mathrm{RC}: 82508$

Disponível em: https://www.nucleodoconhecimento.com.br/gesundheit/kawasakikrankheit 


\section{WIE DIE HAUPTORGANE UND ANATOMISCHEN STRUKTUREN VON DK BEEINFLUSST WERDEN}

DK betrifft Organe und Arterien, vor allem mittelgroße Gefäße, wie die des Herzens, insbesondere koronare Arterien, mit der Bildung von Aneurysmen. Die Krankheit kann auch das Atmungssystem (Lunge), durch Vaskulitis, das Magen-Darm-System, vor allem den Darm und die Gallenblase, und das Nervensystem (CASTRO; URBANO; COSTA, 2009).

Das Lymphsystem kann mit DK betroffen sein, da eines der Symptome von DK die Schwellung der zervikalen Lymphknoten ist. Das Hautsystem wird durch kutanes Erythem beschädigt, gefolgt von Ödemen. In den Augen kann eine nicht-suputive Konjunktivhyperämie auftreten und die Schleimhäute der Mundhöhle können durch Mundschleimhautundung und Läsionen an den Lippen beeinflusst werden (SOTELO; GONZÁLEZ, 2007).

\section{DIE HAUPTMERKMALE DES KLINISCHEN BILDES EINER PERSON MIT DK UND WIE SIE IHN BEEINFLUSSEN}

Der klinische Verlauf von DK kann in drei klinische Stadien unterteilt werden: akute, subakute und Rekonvaleszenz. Das akute febrile Stadium dauert in der Regel von 7 bis 14 Tagen, gekennzeichnet durch Bindehautverstopfung, Mundschleimhaut, Erythem, Abplatzen und polymorphen Hautausschlag und Lymphadenopathie. Das subakute Stadium ist der Zeitraum zwischen dem Ende des Fiebers und dem 25. Tag der Krankheit. In diesem Stadium, Patienten mit Hautabblättern in den Gliedmaßen, Arthritis, Arthralgie und Thrombozytose vorhanden. Das Rekonvaleszenzstadium beginnt, wenn die klinischen Symptome zu verschwinden beginnen, und setzt sich bis zur normalen Etablierung von VHS (roter Blutdruck), der in der Regel 6 bis 8 Wochen nach dem Auftreten der Symptome beobachtet wird, fort. Solche Stadien beeinflussen das Leben des Patienten, da sie inn daran hindern,

RC: 82508

Disponível em: https://www.nucleodoconhecimento.com.br/gesundheit/kawasakikrankheit 
seine täglichen Aktivitäten auszuführen, was hauptsächlich auf Hautläsionen und Symptomatologie zurückzuführen ist (ALMEIDA, 2017; SCARDINA et al., 2007).

Der Zustand, der auf die Pathologie hindeutet, ist durch Fieber, erhöhte zervikale Lymphknoten und Tonsillitis gekennzeichnet, gefolgt von generalisiertem kutanem Erythem, Juckreiz und Ödemen der Gliedmaßen und nach einigen Tagen des Abplatzens. Labortests zeigen eine aktive Entzündliche Phase mit hoher Sedimentationsrate, C-reaktivem Protein, Leukozytose und erhöhter Anzahl von Blutplättchen, was zu Lethargie des betroffenen Individuums führt, da Entzündungen starke Schmerzen verursachen können (ALMEIDA, 2018; ATIK; FORONDA; BUSTAMANTE, 2003; FERRONATO et al., 2010; ROSSI et al., 2015).

Nicht-suputive Konjunt-Hyperämie kann auch auftreten; Makula-Papulularausschlag; Läsionen auf den Lippen und Mundhöhle; Veränderungen in der Haut der Extremitäten, zusätzlich zu präcordialem Murmeln, Atembeschwerden, Bauchschmerzen, Dysurie, Hepatomegalie, Arthralgie, Versteifung des Halses und Krämpfen. Diese Symptome verhindern, dass der Patient sieht und sich normal fortbewegt, da die Augen geschädigt sind und der Schmerz Schwierigkeiten verursacht, Bewegungen durchzuführen (CASTRO; URBANO; COSTA, 2009; SOTELO; GONZÁLEZ, 2007).

DK betrifft mehrere Patienten mit Vaskulitis in verschiedenen Organen, wie bereits gesagt wurde, die Lunge, Darm, Gallenblase, zentrales Nervensystem, was zum Auftreten von Symptomen wie Müdigkeit, Gewichtsverlust und Schmerzen in Gelenken und Muskeln führt, aber Herzschäden ist die bedeutendste, mit der Bildung von koronaren Aneurysmen, die zu Angina führen (CASTRO; URBANO; COSTA, 2009; PRINTZ, 2011).

Ein weiteres Symptom ist Eine Reizung, die bei Kindern mit der Krankheit auftritt, und Magen-Darm-Beschwerden, einschließlich Durchfall, Erbrechen und Bauchschmerzen, treten bei etwa einem Drittel der Patienten auf, was erklärt, dass

$\mathrm{RC}: 82508$

Disponível em: https://www.nucleodoconhecimento.com.br/gesundheit/kawasakikrankheit 
Kinder mit DK mehr gereizt sind als die anderen (ALMEIDA, 2018; KAYIRAN; DINDAR; GURAKAN, 2010).

Abszess und retropharyngeale Ödeme ist in der Regel mit einem tödlichen Zustand verbunden, wenn nicht richtig behandelt. Otorhinolaryngologen können es mit chirurgischer Drainage des Abszesses behandeln, verhindert Atemwegsblock (KIM; KWON, 2016; XIE et al., 2018).

Die Mundschleimhautwirdin wird durch diffuses Erythem der Schleimhaut, Rötung in den Lippen und Zunge und Hypertrophie der lingualen Papille mit anschließender Entwicklung der Erdbeerzunge dargestellt und tritt in der Regel im akuten Stadium der Krankheit und Rekonvaleszenz als Folge der pharmakologischen Behandlung auf und verhindert, dass der Patient aufgrund der Läsionen ordnungsgemäß füttert, was seine adäquate Ernährung beeinträchtigt (SCARDINA et al., 2007).

\section{DIE DIAGNOSIS VON DK}

DK stellt eine Herausforderung dar, frühzeitig diagnostiziert zu werden, da es sich um eine ungewöhnliche rheumatologische Erkrankung handelt, die eine spezifische Intervention erfordert, um schwere oder tödliche Folgen zu vermeiden (ALMEIDA et al., 2010).

Die Klinik der Krankheit ist unklar, da häufig die Erstdiagnose von anderen Krankheiten, wie Scharlach, virales Exantheme oder allergische Erkrankungen, aufgrund der ersten Symptome der Krankheit. Auch bei vollständiger Erfüllung der Kriterien, ist es häufig, die richtige Diagnose zu verzögern, was zu einer Verzögerung am Beginn der entsprechenden Behandlung führt, begünstigt das Auftreten von Komplikationen, die schnell den klinischen Zustand des Patienten verschlimmern können. Darüber hinaus gibt es unvollständige oder atypische Formen, die die Diagnose noch schwieriger machen. Eine frühzeitige Diagnose der Krankheit ist wichtig, da die Behandlung in der akuten Phase den Entzündungsprozess in den

RC: 82508

Disponível em: https://www.nucleodoconhecimento.com.br/gesundheit/kawasakikrankheit 
Herzkranzgefäßen reduziert und Thrombose verhindert, wodurch die Sterblichkeitschancen verringert werden (FERRONATO et al., 2010).

Eine rechtzeitige klinische Diagnose ist unerlässlich, um diese frühe Behandlung zu etablieren und koronare Läsionen zu vermeiden. Diese Art von Verletzung kann Ischämie oder Myokardinfarkt verursachen, die extrem schwer ist (KAYIRAN; DINDAR; GURAKAN, 2010; SOTELO; GONZÁLEZ, 2007).

Die diagnostischen Kriterien der klassischen DK-Krankheit sind Fieber mit fünf Tagen oder mehr, zusätzlich zu mindestens vier anderen Kriterien, die sind: palmoplantare erythem mit oder ohne schmerzhafte Ödeme oder periunigueal abplatzen; Polymorphes Exathem; bulbar konjunktivale Hyperämie auf beiden Seiten, nicht exsudativ; Veränderungen in den Lippen und Mundschleimhaut; Zervikale Lymphadenopathie - Durchmesser größer als $1,5 \mathrm{~cm}$, hart und schmerzlos bei Palpation. Die Diagnose unvollständiger DK kann in Gegenwart von drei oder mehr der folgenden Kriterien festgestellt werden: Albumin $\leq 3 \mathrm{~g} / \mathrm{dL}$; Anämie; Erhöhung der Alanin-Aminotransferase; Thrombozyten nach sieben Tagen $\geq 450.000 / \mathrm{mm}^{3}$; Leukozyten $\geq 15.000 / \mathrm{mm}^{3}$; Urin $\geq 10$ Leukozyten (CASTRO; URBAN; COSTA, 2009; RODRIGUES et al., 2017; YAP; LIN; WANGUI, 2012).

Die Hauptfolgen der Krankheit beziehen sich auf das Herzsystem, daher ist die Kardiographie durch Echokardiographie ein relevanter Teil der Bewertung von Patienten mit Verdacht auf DK. Bei unkomplizierten Fällen wird die Echokardiographie bei der Diagnose empfohlen, 6 bis 8 Wochen nach Beginn der Krankheit (ATIK et al., 2017; KAYIRAN; DINDAR; GURAKAN, 2010)

Die häufigsten Laborveränderungen sind blutbildende Untersuchungen mit Leukozytose und Neutrophilia, zusätzlich zur Erhöhung der Werte von akuten Phasentests, wie Hämosedimentationsgeschwindigkeit und C-reaktives Protein. Die Krankheit wird oft erst nach koronaren Herzleiden diagnostiziert (FERRONATO et al., 2010).

$\mathrm{RC}: 82508$

Disponível em: https://www.nucleodoconhecimento.com.br/gesundheit/kawasakikrankheit 
Eine Studie von Kentsis et al. (2013) fand heraus, dass Filamin-C- und Meprina-AMarker sowohl im Serum als auch im Urin der beiden unabhängigen Gruppen der teilnehmenden DK-Patienten nachgewiesen wurden. Die gleiche Studie stellt fest, dass die Verwendung dieser Marker, um die Diagnose von DK durchzuführen, effizienter ist als die Verwendung von Markern, die derzeit verwendet werden (KENTSIS et al., 2013).

\section{IMAGE EXAMEN UND KOMPLEMENTÄRE}

Echokardiogramm ist einer der wichtigsten Tests für die Diagnose von DK verwendet, da es nicht invasiv ist, hat eine hohe Empfindlichkeit und Spezifität, um Anomalien in den Herzkranzgefäßen zu analysieren und zu erkennen, das Myokard zu bewerten und das Vorhandensein von Perikarditis zu überprüfen. In dieser Untersuchung können einige Befunde auftreten, die auf eine koronare Arteritis hindeuten, die der Bildung von Aneurysmen wie perivaskulärer Helligkeit, Ekekasien oder Verengung der Herzkranzgefäße vorausgeht. Bei der Analyse der Herzkranzgefäße sollte der Innendurchmesser des Gefäßes bewertet werden; Anzahl, Lage und Typ (ob sakkaganz oder fusiform) von Aneurysmen; und das Vorhandensein oder Nichtvorhandensein von intraluminalem Thrombus und Susthese. In der akuten Phase der Krankheit, einige häufige Befunde in Echokardiogrammen sind: Verringerung der linken Ventrikel Kontraktilität, Regurgitation der Mitralklappe und Perikardialerguss (CASTRO; URBANO; COSTA, 2009; MAGALHÃES; ALVES, 2017; RODRIGUES et al., 2017).

Andere Tests, die durchgeführt werden können, basierend auf Verfügbarkeit, Schweregrad, Vorteilen und Indikationen, die je nach Fall variieren. Zum Beispiel Magnetresonanztomographie (RNM), Angioresonanz, Bruströntgen, hochauflösende Computertomographie, Angiographie (CASTRO; URBANO; COSTA, 2009).

CT-Angiographie, eine invasive Untersuchung mit Risiken und Angioresonanz werden in der Regel bei Patienten durchgeführt, die Anomalien auf

RC: 82508

Disponível em: https://www.nucleodoconhecimento.com.br/gesundheit/kawasaki- 
Elektrokardiogramm, Echokardiographie oder Symptome der Myokardischämie (CASTRO; URBANO; COSTA, 2009; MAGALHÃES; ALVES, 2017).

In letzter Zeit wurde die hochauflösende Computertomographie stärker angedeutet, insbesondere im Vergleich zur MRT, da ihre Ausführung schneller und einfacher zu interpretieren ist, während die MRT eine längere Anästhesiezeit von Kindern zur Abholung erfordert (CASTRO; URBANO; COSTA, 2009).

Brust-Röntgen, die in der Regel keine Veränderungen präsentiert, in etwa 15\% der Fälle Anomalien entdeckt werden, wie: Muster der retikulatoranlen oder peribronchialen Infiltrat, Ansammlung von zu viel Flüssigkeit im Raum zwischen der viszeralen und parietalen Pleuras oder Zusammenbruch der Lunge. Sie erscheinen nach dem 10. Tag der Krankheit und sind 10 bis 50 Tage nach Beginn der Erkrankung möglich, Röntgenaufnahmen zu machen (CASTRO; URBANO; COSTA, 2009).

In Labortests sind die Ergebnisse nicht spezifisch für DK, sondern ein Hinweis auf einen hochentzündlichen Prozess und es ist möglich, zu Beginn der akuten Phase eine Erhöhung der Hämosedimentationsgeschwindigkeit (VHS), C-reaktives Protein (PCR) und Säure-Alphaglykoproteinraten zu finden. Im Bluttest ist es möglich, eine ungewöhnlich hohe Menge an Neutrophilen zu überprüfen, genannt Leukozytose mit Neutrophilia, und kann von erhöhten Fledermäusen oder Eosinophilie begleitet werden. Darüber hinaus ist die Menge der Thrombozyten in der akuten Phase in der Regel normal, jedoch ist es möglich, dass in einigen Fällen gibt es Thrombozytopenie, die mit der Entwicklung von schweren koronaren Erkrankungen und Myokardinfarkt verbunden ist (MAGALHÃES; ALVES, 2017).

\section{ANALYSE DER MAIN COMPLICATIONS ASSOCIATED MIT DK}

Die schwerwiegendste Komplikation der Krankheit ist die koronare Vaskulitis, von der 15-20\% der unbehandelten Patienten betroffen sind, was zu Aneurysmen, Ekekasien

RC: 82508

Disponível em: https://www.nucleodoconhecimento.com.br/gesundheit/kawasakikrankheit 
und Susthesis der Herzkranzgefäße führen kann, die für $2 \%$ Sterblichkeit verantwortlich sind. Andere Komplikationen von DK wurden als Aneurysmen in anderen Arterien wie der Aorta beschrieben; die Bauchaorta; Achselhöhle; brachiocephalische Arterie; Iliasarterien, Oberschenkelarterien und Nierenarterien, was darauf hinweist, wie DK den menschlichen Körper stark beeinflussen kann, und damit extrem gefährlich sein kann (ALVES, et al., 2011; CHBEIR et al., 2018; MUTLUER; ÇELIKER, 2019; PRINTZ, 2011; XIE et al., 2018).

Einige Patienten mit DK sind anfällig für progressive koronare Dilatation. Eine solche koronare Arteriendilatation tritt während der akuten Phase auf und kann in die subakute Phase übergehen, die den klinischen Zustand mehrerer Patienten verschlimmert (BRIEDÉ et al., 2015; 2015; LIU et al., 2017).

Magen-Darm-Komplikationen bei DK können auftreten, wie Darmverstopfung, Dickdarmödem, Darm-Ischämie, Darm-Pseudo-Obstruktion, und akuter Bauch, der den Patienten alarmierend betrifft, da diese Komplikationen zu Sepsis und sogar zum Tod führen können. Darüber hinaus können ophthalmologische Veränderungen im Zusammenhang mit DK Uveitis, Iridocyclitis, Bindehautblutung, optische Neuritis, Amaurose und Obstruktion der Augenarterie, die irreparable Fortsetzungen mehrmals verursacht. DK manifestiert sich auch als nekrotisierende Vaskulitis, was zum Verlust des betroffenen Gewebes führt (ALVES, et al., 2011).

Neurologische Komplikationen aufgrund einer Verletzung des Zentralnervensystems (SNC) können wie folgt sein: Meningoenzephalitis, subdurale Sammlung, zerebrale Hypoperfusion, Ischämie, zerebraler Infarkt, Anfälle, Emiplegie, geistige Verwirrung, Lethargie und sogar Koma oder zerebraler Infarkt. Solche Komplikationen können irreversibel werden und das Leben des Patienten dauerhaft schädigen (ALVES, et al., 2011).

Das Risiko von Spätkomplikationen von DK deutet darauf hin, dass neben der Kindheit auch eine langfristige Nachsorge erforderlich ist (GIACCHI et al., 2014).

$\mathrm{RC}: 82508$

Disponível em: https://www.nucleodoconhecimento.com.br/gesundheit/kawasakikrankheit 


\section{BEHANDLUNGEN VERFÜGBAR FÜR INDIVIDUALS AFFECTED VON DK}

Die am meisten indizierte Behandlung ist die Anwendung von IVIG (Intravenöses Immunglobulin) in einer Einzeldosis von $2 \mathrm{~g} / \mathrm{kg}$ pro 12-stündige rinfusion. Nach der Entwicklung des klinischen Quaros des Patienten kann die Dosis von IVIG wiederholt oder Kortikosteroide hinzugefügt werden, insbesondere in feuerfesten Fällen. Andere Medikamente werden empfohlen, wie Cyclophosphamid, Cyclosporin, und Ulinastatin. Außerdem wird ein Produkt auf der Grundlage monoklonaler Antikörper gegen Tumornekrosefaktor alpha sowie ein monoklonaler Antikörper, bekannt als Abciximab, empfohlen, der Aneurysmen schneller behandeln kann (ATIK, 2007; FERRONATO et al., 1010; KAYIRAN; DINDAR; GURAKAN, 2010; PHILIP et al., 2017; SOTELO; GONZÁLEZ, 2007).

Etwa 10 bis 15\% der Patienten mit DK zeigen keine Verbesserung mit der IGIVBehandlung, der Standardbehandlung (MEHNDIRATTA et al., 2014). Bei pädiatrischen Patienten mit DK bieten hohe Dosen von Acetylsalicylsäure (AAS) eine entzündungshemmende Wirkung, während niedrige Dosen eine hemmende Wirkung der Thrombozytenaggregation haben. So wird in der akuten Phase der Krankheit AAS $80-100 \mathrm{mg} / \mathrm{kg} / \mathrm{Tag}$ verwendet, die im Laufe des Tages in vier Einnahmeungen unterteilt ist, um die entzündungshemmende Wirkung von IGIV zu verstärken. Während in einigen Krankenhäusern die Dosis von AAS nach 2-3 Tagen in Abwesenheit von Fieber reduziert wird, dauert die Behandlung in anderen Gesundheitszentren bis zu 2 Wochen an. Darüber hinaus haben Kinder mit Herzanomalien ein Rezept für das Unbestimmtseinhören (ATIK, 2007; CASTRO; URBANO; COSTA, 2009; MEHNDIRATTA et al., 2014).

Immunsuppressive Medikamente wie Cyclophosphamid werden auch zusammen mit Prednison oder Methylprednisolon verwendet, um einige Symptome wie Fieber zu

RC: 82508

Disponível em: https://www.nucleodoconhecimento.com.br/gesundheit/kawasakikrankheit 
verbessern, aber diese Medikamente haben keine therapeutische Wirkung auf Herzanomalien (CASTRO; URBANO; COSTA, 2009; PHILIP et al., 2017).

In der akuten Phase der DK-Erkrankung besteht ein höheres chirurgisches Risiko, da bei Einer Entzündung anastomosis mit entzündeter Herzkranzgefäße das Risiko einer Obstruktion besteht. Der chirurgische Ansatz sollte sich auf Patienten mit riesigen Aneurysmen oder ischämischen Prozessen nach der akuten Phase beschränken (ATIK, 2007; SOTELO; GONZÁLEZ, 2007).

Die chirurgische Revaskularisation von koronaren Läsionen, die für DK sekundär sind, ist relativ selten. Nach den aktuellen Richtlinien sollte eine vollständige arterielle Revaskularisation bei jungen Patienten mit kleinen Komorbiditäten angewendet werden, um eine gute langfristige Transplantatdurchlässigkeit zu erhalten (BARCA et al., 2019).

Zusätzlich zur Myokardrevaskularisationschirurgie, die die Behandlung der Wahl bei thrombotischen Koronarkomplikationen bei DK war, perkutane Koronarintervention (ICP) mit regulären oder perkutanen Stents mit Polytetrafluorethylen (PTFE), Spulenimplantation, perkutane transluminale Koronarrevaskularisation (PTCR) mit Anwendung von Thrombolytika in der infarktbedingten Arterie und perkutane Koronarrotationsablation (PTCRA) wurden bei von der Krankheit betroffenen Patienten angewendet (MUTLUER; ÇELIKER, 2019).

Was das Anästhesiemanagement betrifft, so gibt es wenig Informationen beschrieben, jedoch, Myokardrevaskularisation mit Anästhesie durch halogenierte Gase durchgeführt weniger Schäden, so zeigt eine bessere postoperative Erholung der Myokardfunktion im Vergleich zu intravenösen Anästhetika. So begünstigt es die Hemmung der neuroendokrinen Reaktion auf Stress, und bereitet den Patienten auch auf eine frühe Extubation in der postoperativen Periode, die bei der Prävention von möglichen Komplikationen durch Intubation hilft (MARTíNEZ; MÉNDEZ, 2013).

RC: 82508

Disponível em: https://www.nucleodoconhecimento.com.br/gesundheit/kawasakikrankheit 


\section{SCHLUSSFOLGERUNG}

Das Vorkommen von DK ist variabel, da es in der Region Asien präsenter ist, insbesondere bei Kindern unter 5 Jahren. In Lateinamerika ist die Inzidenz seltener und die Sterblichkeit dieser Pathologie ist gering.

Der ätiologische Wirkstoff von DK wurde nicht identifiziert, aber es gibt einige Krankheitserreger, die mit dieser Krankheit zusammenhängen können. Es gibt Hinweise darauf, dass diese Krankheit eine genetische Beziehung hat, dies wurde aufgrund der erhöhten Inzidenz unter asiatischen Menschen und unter Verwandten von Patienten, die von DK betroffen sind, gefunden, darüber hinaus gibt es auch einige Gene, die mit dieser Pathologie verbunden sind.

Es gibt Veränderungen im Zusammenhang mit DK wie systemische Entzündungen, die bestimmte Organe durch Angiitis, sterile Meningitis, Pneumonitis, Adenofinit und Hepatitis verursacht beeinflussen. DK betrifft Organe und Arterien, vor allem mittelkalibrige Gefäße, wie die des Herzens, so dass zur Bildung von Aneurysmen führt, die Augen, Schleimhäute der Mundhöhle, die Lunge, den Darm, die Gallenblase und das Nervensystem, das Lymphsystem, das Hautsystem und Läsionen in den Lippen beeinflussen können.

Das klinische Bild von DK hat drei Stadien. Das akute febrile Stadium ist durch Bindehautverstopfung, Mundschleimhautitis, Erythem, Abplatzen, polymorphen Hautausschlag und laterozeratrikale Lymphadenopathie gekennzeichnet. Das subakute Stadium tritt am Ende des Fiebers auf, Patienten haben Hautabblättern an den Gliedmaßen, Arthritis, Arthralgie und Thrombozytose. Das Rekonvaleszenzstadium entsteht, wenn die Symptome fast verschwinden und sich bis zu ihrer Normalisierung fortsetzt.

Die Verzögerung bei der korrekten Diagnose von DK ist recht häufig, was zu einer Verzögerung beim Beginn der Behandlung führen kann, was zu Komplikationen

$\mathrm{RC}: 82508$

Disponível em: https://www.nucleodoconhecimento.com.br/gesundheit/kawasakikrankheit 
führen kann, die den klinischen Zustand verschlimmern. Eine frühzeitige klinische Diagnose ist äußerst wichtig, um eine frühzeitige Behandlung zu etablieren und koronare Arterienverletzungen zu vermeiden.

Die Behandlung von DK basiert auf der Entwicklung des klinischen Bildes des Patienten. Die am häufigsten verwendete Therapie ist die Anwendung von IGIV, wenn Veränderungen in der Klinik auftreten, kann die Dosis von IGIV wiederholt werden oder Kortikosteroide können hinzugefügt werden. Andere Arzneimittel können indiziert werden, wie Cyclophosphamid, Cyclosporin und Ulinastatin. Es wird auch ein Produkt auf der Grundlage monoklonaler Antikörper Infliximab, Immunsuppressiva wie Cyclophosphamid oder Cyclosporin und Prednison oder Methylprednisolon verwendet werden. Die chirurgische Behandlung erfolgt hauptsächlich in der akuten Phase von DK, sollte aber nach der akuten Phase der Krankheit auf Patienten mit riesigen Aneurysmen oder ischämischen Prozessen beschränkt werden.

\section{VERWEISE}

ALMEIDA, F. C. Avaliação das manifestações clínicas e achados laboratoriais em 301 pacientes com doença de Kawasaki: acompanhamento de dez anos. Tese (Doutorado em Ciências Médicas). Faculdade de Medicina, Universidade de Brasília, Brasília. 2018.

ALMEIDA, M. A. A. L. S. Avaliação da calprotectina e dos anticorpos anticitoplasma de neutrófilos como marcadores de inflamação e autoimunidade nas diferentes fases da doença de Kawasaki. Dissertação (Mestrado em Ciências Farmacêuticas). Faculdade de Ciências da Saúde, Universidade de Brasília, Brasília. 2017.

$\mathrm{RC}: 82508$

Disponível em: https://www.nucleodoconhecimento.com.br/gesundheit/kawasakikrankheit 
ALMEIDA, R. G. et al. Perfil da doença de Kawasaki em crianças encaminhadas para dois serviços de reumatologia pediátrica do Rio de Janeiro, Brasil. Revista Brasileira de Reumatologia, v. 50, n. 5, p. 529-538, set./out. 2010.

ALVES, N. R. M. et al. Estudo prospectivo das complicações da Doença de Kawasaki: análise de 115 casos. Revista da Associação Médica Brasileira, v. 57, n. 3, p. 299-305, mar. 2011.

ATIK, E. Doença de Kawasaki: Regressão de Aneurismas Gigantes das Artérias Coronárias com Obstrução Tardia Posterior Kawasaki Disease: Giant Coronary Arteries Aneurysms Regression and Later Stenosis. Arquivos brasileiros de Cardiologia, v. 88, n. 1, p. 22-23, 2007.

ATIK, E.; FORONDA, A.; BUSTAMANTE, L. N. P. Kawasaki Disease. Involution of Giant Coronary Aneurysms After Prolonged Anti-inflammatory Treatment. Arquivos brasileiros de Cardiologia, v. 81, n. 3, p. 265-72, set. 2003.

ATIK, E. et al. Caso 6/2017 - Extenso Aneurisma Gigante de Artéria Coronária Esquerda por Vasculite de Kawasaki em Homem Assintomático com 48 Anos de Idade. Arquivos brasileiros de Cardiologia, v. 109, n. 5, p. 489-490, nov. 2017.

BARCA, L. V. et al. Ligature of the Left Main Coronary Artery after Surgery in Kawasaki Disease: Case Report. Braz J Cardiovasc Surg, v. 34, n. 1, p. 111-113, jan./fev., 2019.

BRIEDÉ, S. et al. Hamoen M, Oosterveld M JS, Breur JMPJ. Langetermijneffecten van de ziekte van Kawasaki. Ned tijdschr geneeskd, v. 154, n. 2121, p. 1-6, out. 2015.

CASTRO, P. A.; URBANO, L. M. F.; COSTA, I. M. C. Doença de Kawasaki. Anais Brasileiros de Dermatologia, v. 84, n. 4, p. 317-331, mai. 2009.

$\mathrm{RC}: 82508$

Disponível em: https://www.nucleodoconhecimento.com.br/gesundheit/kawasakikrankheit 
CHBEIR, D. et al. Kawasaki disease: abnormal initial echocardiogram is associated with resistance to IV Ig and development of coronary artery lesions. Pediatric Rheumatology Onlinw Journal, v. 6, n. 48, p. 1-10, jul. 2018.

FERRONATO, A. E. et al. Doença de Kawasaki: experiência clínica em hospital universitário. Revista Paulista de Pediatria, v. 28, n. 2, p. 148-154, jun. 2010.

GIACCHI, V. et al. Avaliação do espessamento íntimo da artéria coronária em pacientes com diagnóstico prévio de doença de Kawasaki por meio de ecocardiografia transtorácica de alta resolução: nossa experiência. BMC Cardiovasc Disord, v. 14, n. 106, p 1-6, ago. 2014.

HUANG, X. et al. Influenza infection and Kawasaki disease. Revista da Sociedade Brasileira de Medicina Tropical, v. 48, n. 3, p. 243-248, jun. 2015.

KAYIRAN, S. M.; DINDAR, A. N.; GURAKAN, B. An evaluation of children with Kawasaki disease in Istanbul: a retrospective follow-up study. Clinical Science, v. 65, n. 12, p. 1261-1265, dez. 2010.

KENTSIS, A. et al. Urine proteomics for discovery of improved diagnostic markers of Kawasaki disease. EMBO Mol Med, v. 5, n. 2, p. 210-220, fev. 2013.

KIM, J. S.; KWON, S. H. Atypical Kawasaki disease presenting a retropharyngeal abscess. Braz J Otorhinolaryngol, v. 82, n. 4, p. 484-486, 2016.

LIU, M. Y. et al. Risk factors and implications of progressive coronary dilatation in children with Kawasaki disease. BMC Pediatrics, v. 17, n. 139, p. 1-7, jun. 2017.

MAGALHÃES, C. M. E. Estudo da prevalência da perda auditiva neurossensorial como complicação da doença de Kawasaki. Tese (Doutorado em Ciências da Saúde) - Faculdade de ciências da saúde, Universidade Federal de Brasília, Brasília. 2008.

$\mathrm{RC}: 82508$

Disponível em: https://www.nucleodoconhecimento.com.br/gesundheit/kawasakikrankheit 
MAGALHÃES, C. M. R.; ALVES, N. R. M. Doença de Kawasaki. In: I. Burns, DAR, Campos Júnior D, Silva LR, Borges WG. Tratado de pediatria: Sociedade Brasileira de Pediatria. 4. ed. Barueri, SP: Manole, 2017. p.1825-1835.

MARTÍNEZ, U. M.; MÉNDEZ, F. M. Manejo anestésico del paciente com enfermedad de Kawasaki durante la cirugía de revascularización coronaria: informe de um caso. Archivos de Cardiología de México, v. 83, n. 4, p. 267-272, abr. 2013.

MEHNDIRATTA, S. et al. A case of incomplete and refractory Kawasaki disease: Diagnostic and therapeutic challenges. The South African Journal of Child Health, v. 8, n. 1, p. 37-38, fev. 2014.

MUTLUER, F. O.; ÇELIKER, A. Comment on Ligature of the Left Main Coeonary Artery after Surgery in Kawasaki Disease: Case Report. Brazilian Journal of Cardiovascular Surgery, v. 34, n.3, p. 382, jul. 2019.

PHILIP, S. et al. Role of Antioxidants in Horse Serummediated Vasculitis in Swine: Potential Relevance to Early Treatment in Mitigation of Coronary Arteritis in Kawasaki Disease. Pediatrics and Neonatology, v. 58, n. 4, p. 328-337, fev. 2017.

PRINTZ, B. F. Noncoronary Cardiac Abnormalities Are Associated With Coronary Artery Dilation and With Laboratory Inflammatory Markers in Acute Kawasaki Disease. Journal of the American College of Cardiology, v. 57, n. 1, p. 68-92, jan. 2011.

RODRIGUES, M. et al. Doença de Kawasaki e Complicações Cardiovasculares em Pediatria. Birth and growth medical jornal, v.27, n. 1, p. 54-58, fev. 2017.

ROSSI, F. S. et al. Extensa linfadenite cervical mimetizando adenite bacteriana como primeira manifestação da doença de Kawasaki. Einstein, v. 13, n. 3, p. 426429, jul./set. 2015.

$\mathrm{RC}: 82508$

Disponível em: https://www.nucleodoconhecimento.com.br/gesundheit/kawasakikrankheit 
ROWLEY, A. H. et al. Allograft Inflammatory Factor-1 Links T-Cell Activation, Interferon Response, and Macrophage Activation in Chronic Kawasaki Disease Arteritis. Journal of the Pediatric Infectious Diseases Society, v. 6, n. 3, p. 94102, set. 2017.

SCARDINA, G. A. et al. Oral necrotizing microvasculitis in a patient affected by Kawasaki disease. Medicina Oral Patologia Oral y Cirugia Bucal, v. 12, n. 8, p. 560-564, dez. 2007.

SOTELO, N.; GONZÁLEZ, L. A. Kawasaki disease: A rare pediatric pathology in Mexico Twenty cases report from the Hospital Infantil del Estado de Sonora. Archivos de Cardiologia de Mexico, v. 77, n. 4, p. 299-307, dez. 2007.

XIE, X. F. et al. Proteomics study of serum exosomes in Kawasaki disease patients with coronary artery aneurysms. Cardiology Journal, v. 26, n. 5, p. 584-593, fev. 2018.

YAP, C. Y.; LIN, L. H.; WANGI, N. K. An atypical presentation of Kawasaki disease: a 10- year-old boy with acute exudative tonsillitis and bilateral cervical lymphadenitis. CLINICS, v. 67, n. 6, p. 689-692, 2012.

Eingereicht: April 2021.

Genehmigt: April 2021.

RC: 82508

Disponível em: https://www.nucleodoconhecimento.com.br/gesundheit/kawasakikrankheit 